\title{
PERSPECTIVE
}

\section{FORESTRY EDUCATION}

As this is the special edition on education, my perspective is devoted to a discussion of some of the forestry education issues in the news today. First and foremost, there has been a lot of talk about the declining enrolment in forestry programs across the country. Universities and colleges seem to be suffering equally from this problem. Various recruiting strategies are being discussed to address the issue and I am sure you have heard most of reasons being offered up as the root cause of the problem. The cost of post-secondary education, especially associated with university, gets quite a bit of credit in this regard. But would that not result in other program enrolment numbers being down as well? Would it not also result in a shift to the colleges where tuition and other costs are not as intimidating? This cost agrument includes references to government cutbacks and the dwindling support for education that is driving tuition and fees higher and higher at unprecedented rates. I do not totally rule out the cost argument. However, it alone does not answer the question.

Another popular view focuses on the shift to urban living, with all the amenities associated with this life style close at hand. Linked to this idea is the thought that the great outdoors is for recreational purposes. As such, working in the

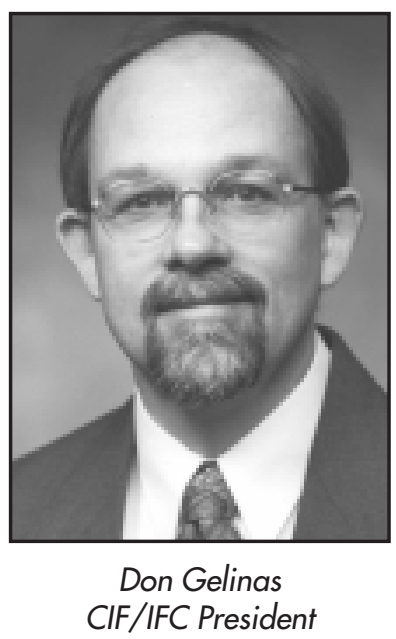

\section{EDUCATION FORESTIER}

Compte tenu qu'il s'agit d'une édition spéciale sur l'éducation, cette perspective est consacrée à discuter de quelques-uns des enjeux retrouvés dans l'actualité. Premièrement et le plus important, il y a beaucoup de discussions au sujet des inscriptions à la baisse dans les programmes de foresterie de tout le pays. Les universités et les collèges semblent souffrir tous deux de ce problème. Diverses stratégies de recrutement sont discutées pour faire face à ce problème, et je suis certain que vous avez eu vent de la plupart des raisons avancées comme étant la source du problème. Le coût de l'éducation post-secondaire, spécialement celle associée à la formation universitaire, reçoit une partie importante de l'intérêt dans ce domaine. Mais est-ce que cela n'entraînerait pas également une réduction des inscriptions dans d'autres programmes ? Est-ce que cela ne résulterait pas en un revirement vers les collèges où les frais de scolarité et les autres coûts ne sont pas aussi intimidants ? L'argument relié aux coûts fait également référence aux coupures gouvernementales et aie la réduction de l'aide aie l'éducation qui font en sorte que les frais de scolarité et d'études sont de plus en plus élevés et atteignant un niveau sans précédent. Je ne mets pas totalement de côté l'argument monétaire, mais il ne répond pas seul à la question.

Un autre aspect courant se concentre sur la migration vers les milieux urbains, avec la proximité de toutes les commodités associées à ce type de vie. L'idée sous-jacente est la pensée que les grands espaces sont destinés aux loisirs. En tant que tel, le travail en forêt, particulièrement en hiver, a perdu de son attirance. Vous pouvez utiliser cet argument à un niveau supérieur et vous attarder sur l'accroissement de la sensibilisation du public en matière d'environnement et de la perception publique que la foresterie dégage un aspect négatif. Qu'il s'agisse d'arguments portant sur le déboisement ou d'une question sur les habitats fauniques, la foresterie a été la cible de relations publiques dommageables au cours des 10 à 20 dernières années. Cela coïncide à peu de choses près au groupe d'âge que nous devrions nous attendre à voir franchir la porte pour s'inscrire aujourd'hui dans une carrière en foresterie. Estce que ces étudiants sont découragés de nous ? Est-ce qu'ils optent à la place pour des programmes de conservation des ressources environnementales ? Ou est-ce qu'ils abandonnent simplement l'idée d'une formation post-secondaire en environnement?

On a suggéré également que les emplois en foresterie ne sont pas les mieux rémunérés qui existent. Les étudiants disent en conséquence, que s'ils sont pour consacrer près de cinq années de leur vie à une formation post-secondaire, ils devraient au moins le faire dans un domaine bien rémunérateur une fois sur le marché du travail. D'autres considèrent encore qu'ils n'ont pas besoin d'une formation post-secondaire pour occuper un bon poste sur le marché du travail. Pour ceux qui appuient cet argument, les syndicats sont la racine du mal.

Finalement, il y a les supporters de la théorie des phénomènes naturels. Pour ces personnes, tout évolue selon un cycle ou en 
ment - all the good ones have already been spoken for. Well, as is the case with anything else worth pursuing, the answer is not easily arrived at. However, it would help if we didn't waste any time on the things we cannot change; the rising cost of education for example. I am beginning to think that rising costs can be added to the list, with death and taxes, as the only sure things in life! As for higher paying jobs and natural cycles, the first is the stuff dreams are made of and the second belongs in the realm of Mother Nature.

That leaves us with the issue of our public reputation. Here is something we can work on! In fact, there is already much happening that will help to improve the image of forestry in the eyes of the public. In this regard, we must remember that, as forest practitioners, we all have a role to play. We are the best ambassadors for our chosen profession. Isn't it interesting that the solution to our "education" problem is education itself!

\section{Editorial from page 188}

With $96 \%$ of the commercial forest in public ownership, the management challenges and issues are enormous, interesting to students and, in some ways, better funded.

Why am I retired from UBC and still working here for no pay? Because I find forestry interesting and challenging. I enjoy teaching at the Alberta Advanced Forest Management Institute and the Forest Management Institute of BC. I am still learning from the grad students. Life really is continual learning.

Gordon Weetman University of British Columbia d'autres mots, «Ce qui s'en va, reviendra! » En tant que tel, ils disent que nous vivons actuellement la phase négative du cycle naturel des choses et que rien ne peut être fait autrement que de passer à travers cette phase. Dans les faits, ils poursuivent en disant que si nous faisons autre chose que d'observer les phénomènes, nous allons perturber l'ordre naturel des choses... ce qui est d'importance suprême face aux arrosages contre la tordeuse des bourgeons de l'épinette!

Alors, qu'est-ce qu'il faut faire ? Il ne sert à rien de consacrer encore du temps à réfléchir sur les raisons du déclin des inscriptions pour les programmes de foresterie - toutes les raisons valides ayant été déjà explorées. Et bien, comme c'est le cas avec tout ce qui mérite d'être poursuivi, nous sommes loin d'une réponse. Cependant, cela aiderait si nous ne consacrions plus de temps sur les choses que nous ne pouvons pas modifier, la hausse des coûts de l'éducation étant un exemple. Je commence à croire que la hausse des coûts peut être ajoutée à la liste, avec la mort et les impôts, comme la seule certitude de la vie. Pour ce qui est des emplois mieux rémunérés et des cycles naturels, le premier correspond à ce dont est composé les rêves, et le deuxième appartient au royaume de Mère Nature.

Reste alors la question de notre réputation publique. Voilà quelque chose sur laquelle nous pouvons travailler! Dans les faits, il y a beaucoup de choses en voie de réalisation qui aideront à améliorer l'image de la foresterie aux yeux du public. À cet effet, nous devons nous souvenir qu'en tant que praticiens de la foresterie, nous avons tous un rôle à jouer. Nous sommes les meilleurs ambassadeurs de cette profession que nous avons choisie. N'est-il pas intéressant que la solution à notre problème «d'éducation » ne soit pas l'éducation en elle-même ?

\section{Éditorial}

et petit à petit il y a plus d'argent pour alimenter le « système » de formation en foresterie. De plus en plus, les compagnies forestières, et même les ONG environnementales, s'associent aux organismes de financement et aux écoles forestières et aux étudiants gradués pour financer et résoudre les problèmes d'aménagement forestier, de transformation et de commercialisation.

Je crois que l'avenir semble très prometteur pour les étudiants canadiens en foresterie et pour la foresterie au Canada. Il s'agit de la «naissance d'une nouvelle ère ». Compte tenu que $96 \%$ des forêts commerciales sont de propriété publique, les défis et les enjeux en aménagement sont énormes, intéressants pour les étudiants et, d'une certaine façon, mieux financés.

Pourquoi suis-je retraité de l'UBC et encore à l'œuvre ici sans salaire ? Parce que je considère la foresterie intéressante et pleine de défis. J'aime enseigner à l'Institut d'aménagement forestier avancé de l'Alberta et à l'Institut d'aménagement forestier de la C.-B. Je continue d'en apprendre de mes étudiants gradués. La vie est un processus continu d'apprentissage.

Gordon Weetman University of British Columbia 